\title{
Holographic description of heavy-flavored baryonic matter decay involving glueball
}

\author{
Si-wen $\mathrm{Li}^{*}$ \\ Department of Physics, School of Science, Dalian Maritime University, Dalian 116026, China
}

(Received 22 December 2018; published 20 February 2019)

\begin{abstract}
We holographically investigate the decay of the heavy-flavored baryonic hadron involving a glueball by using the Witten-Sakai-Sugimoto model. Since the baryon in this model is recognized as the D4-brane wrapped on $S^{4}$ and the glueball field is identified as the bulk gravitational fluctuations, the interaction of the bulk graviton and the baryon brane could be naturally interpreted as a glueball-baryon interaction through the holography which is nothing but the close-open string interaction in string theory. In order to take the heavy flavor into account, an extra pair of heavy-flavored branes separated from the other flavor branes with a heavy-light open string is embedded into the bulk. Due to the finite separation of the flavor branes, the heavy-light string creates massive multiplets which could be identified as the heavy-light meson fields in this model. As the baryon brane on the other hand could be equivalently described by the instanton configuration on the flavor brane, we solve the equations of motion for the heavy-light fields with the Belavin-Polyakov-Schwarz-Tyupkin (BPST) instanton solution for the $N_{f}=2$ flavored gauge fields. Then, with the solutions, we evaluate the soliton mass by deriving the flavored onshell action in the strong coupling limit and heavy quark limit. After the collectivization and quantization, the quantum mechanical system for the glueball and heavy-flavored baryon is obtained in which the effective Hamiltonian is time dependent. Finally, we use the standard technique for the time-dependent quantum mechanical system to analyze the decay of the heavy-flavored baryon involving the glueball, and we find one of the decay processes might correspond to the decay of the baryonic B-meson involving the glueball candidate $f_{0}(1710)$. This work is a holographic approach to studying the decay of the heavy-flavored hadron in nuclear physics.
\end{abstract}

DOI: 10.1103/PhysRevD.99.046013

\section{INTRODUCTION}

Quantum chromodynamics (QCD) as the fundamental theory of nuclear physics predicts the bound state of pure gluons [1-3] because of its non-Abelian nature. Such a bound state is always named a "glueball," which is believed to be the only possible composite particle state in the pure Yang-Mills theory. In general, glueball states could have various Lorentz structures, e.g., a scalar, pseudoscalar, or a tensor glueball with either normal or exotic $J^{P C}$ assignments. Although the glueball state has not been confirmed by the experiment, its spectrum has been studied by the simulation of lattice QCD [4-6]. According to the lattice calculations, it indicates that the lightest glueball state is a scalar with assignment of $0^{++}$and its mass is around 1500-2000 MeV [4,7]. These results also suggest

\footnotetext{
"siwenli@dlmu.edu.cn
}

Published by the American Physical Society under the terms of the Creative Commons Attribution 4.0 International license. Further distribution of this work must maintain attribution to the author(s) and the published article's title, journal citation, and DOI. Funded by SCOAP ${ }^{3}$. that the scalar meson $f_{0}(1710)$ could be considered as a glueball state. The glueball may be produced by the decay of various hadrons in the heavy-ion collision [8-10], so the dynamics of the glueball is very significant. However, lattice QCD involving real-time quantities is extremely complex, and phenomenological models usually include a large number of parameters with some corresponding uncertainties. Thus, it is still challenging to study the dynamics of glueball with traditional quantum field theory.

Fortunately, there is an alternatively different way to investigate the dynamics of glueball based on the famous AdS/CFT correspondence or the gauge-gravity duality pioneered in [11] where a top-down holographic approach from string theory by Witten [12] and Sakai and Sugimoto [13] (i.e., the WSS model) is employed. Analyzing the AdS/CFT dictionary with the WSS model, the glueball field is identified as the bulk gravitational fluctuations carried by the close strings while the meson states are created by the open strings on the $N_{f}$ probe flavor branes. Hence this model naturally includes the interaction of glueball and meson through the holography which is nothing but the close-open string interaction in string 
TABLE I. The brane configuration of the WSS model: “...” denotes the world volume directions of the D-branes.

\begin{tabular}{|c|c|c|c|c|c|c|c|c|c|c|}
\hline & 0 & 1 & 2 & 3 & (4) & $5(U)$ & 6 & 7 & 8 & 9 \\
\hline Colored $N_{c} \mathrm{D} 4$ & $\cdots$ & $\cdots$ & $\cdots$ & $\cdots$ & $\cdots$ & & & & & \\
\hline Flavored $N_{f} \mathrm{D} 8 / \overline{\mathrm{D} 8}$ & $\cdots$ & $\cdots$ & $\cdots$ & $\ldots$ & & $\cdots$ & $\cdots$ & $\cdots$ & $\cdots$ & $\cdots$ \\
\hline Baryon vertex D4' & $\cdots$ & & & & & & $\ldots$ & $\cdots$ & $\cdots$ & $\ldots$ \\
\hline
\end{tabular}

theory. Along this direction, decay of glueball into mesons has been widely studied with this model e.g., in [14-16]. ${ }^{1}$

Keeping the above information in mind and partly motivated by [8-10], in this work we would like to holographically explore the glueball-baryon interaction particularly involving the heavy flavor as an extension to the previous study in [19]. In the WSS model, baryon is identified as the D4'-brane ${ }^{2}$ wrapped on $S^{4}[13,20]$ (namely the baryon vertex) which could be equivalently described by the instanton configuration on the flavored D8-branes according to the string theory $[21,22]$. The configuration of various D-branes is illustrated in Table I. In order to take into account the heavy flavor, we embed an extra pair of flavored $\mathrm{D} 8 / \overline{\mathrm{D} 8}$-brane into the bulk geometry which is

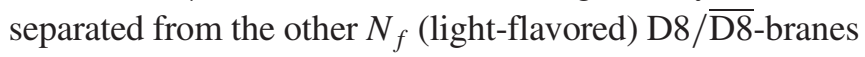
with an open string (the heavy-light string) stretched between them [23,24] as illustrated in Fig. 1. In this configuration, there would be additional multiplets created by the heavy-light (HL) string and they would acquire mass due to the finite separation of the flavor branes. Hence, we could interpret these multiplets as the HL meson fields and the instanton configuration on the D8-branes with the multiplets would include heavy flavor and, thus, can be identified as the heavy-flavored baryon $[25,26]$. So, similarly to the case of the glueball and meson, there must be glueball-baryon interaction in holography as the close string interacting with the D4'-brane carrying the heavy flavor through the HL string or, namely, as the graviton interacting with the heavy-flavored instantons.

Let us outline the content and the organization of this manuscript here. We consider the baryonic bound states created by the baryon vertex in this model with two flavors i.e., $N_{f}=2$. Following [22,24-26], baryons are identified as Skyrmions in the WSS model and they can be described by a quantum mechanical system for their collective modes in the moduli space. The effective Hamiltonian could be obtained by evaluating the classical mass of the soliton $S^{\text {onshel }}=-\int d t M_{\text {soliton. }}$ So the main goal of this paper is to evaluate the effective Hamiltonian involving glueballbaryon interaction with heavy flavor. In Sec. II, we specify the setup with the heavy flavor in this model and solve the classical equations of motion for the HL meson field on the

\footnotetext{
${ }^{1}$ Since the WSS model is based on $\mathrm{AdS}_{7} / \mathrm{CFT}_{6}$ correspondence, several previous work is also relevant to this model e.g., $[17,18]$.

${ }^{2}$ We will use "D4'-brane" to distinguish the baryon brane from those $N_{c}$ D4-branes as color branes throughout the manuscript.
}

flavor brane. In Sec. III, we search for the analytical solutions of the bulk gravitational fluctuations then explicitly compute the onshell action with these solutions. All the calculations are done in the limitation of large 't Hooft coupling constant $\lambda$ where the holography is exactly valid. The final formulas of the effective Hamiltonian depend on the glueball field and the number of heavy-flavored quarks so that it is time dependent. Therefore, the method for the time-dependent system in quantum mechanics would be suitable to describe the decay of heavy-flavored baryons under the classical glueball field. Consequently, we obtain several possible decay processes with the effective Hamiltonian and pick out one of them which might probably correspond to the decay of baryonic B-meson involving the glueball candidate $f_{0}(1710)$ as discussed in $[8-10]$.

Since the WSS model has been presented in many lectures, for reader's convenience we only collect some relevant information about this model in Appendixes A, B, and $C$ which can be also reviewed in [13,22,25-27]. Respectively the gravitational polarization used in this paper are collected in Appendix A. Some useful formulas about the D-brane action and the embedding of the probe branes and string in our setup can be found in Appendix B. In Appendix $\mathrm{C}$, it reviews the effective quantum mechanical system for the collective modes of baryon. At the end of this manuscript, some messy but essential calculations about our main discussion have been summarized in Appendix D.

\section{BARYON AS INSTANTON WITH HEAVY FLAVOR}

The baryon spectrum with pure light flavors in this model is reviewed in Appendix C, so we only outline how to include the heavy flavor in this section. Some necessary information about the embedding of the probe branes and string in our setup could be reviewed in Appendix B.

A simple way to involve the heavy flavor in this model is to embed an extra pair of flavored $\mathrm{D} 8 / \overline{\mathrm{D} 8}$-brane separated from the other $N_{f}$ (light-flavored) D8/ $\overline{\mathrm{D} 8}$-branes with an open string (the heavy-light string) stretched between them as illustrated in Fig. 1. The HL string creates additional multiplets according to the string theory [27] since it connects to the separated branes. And these multiplets could be approximated by local vector fields near the worldvolume of the light flavor branes. Note that the multiplets acquire mass due to the finite length, or namely 


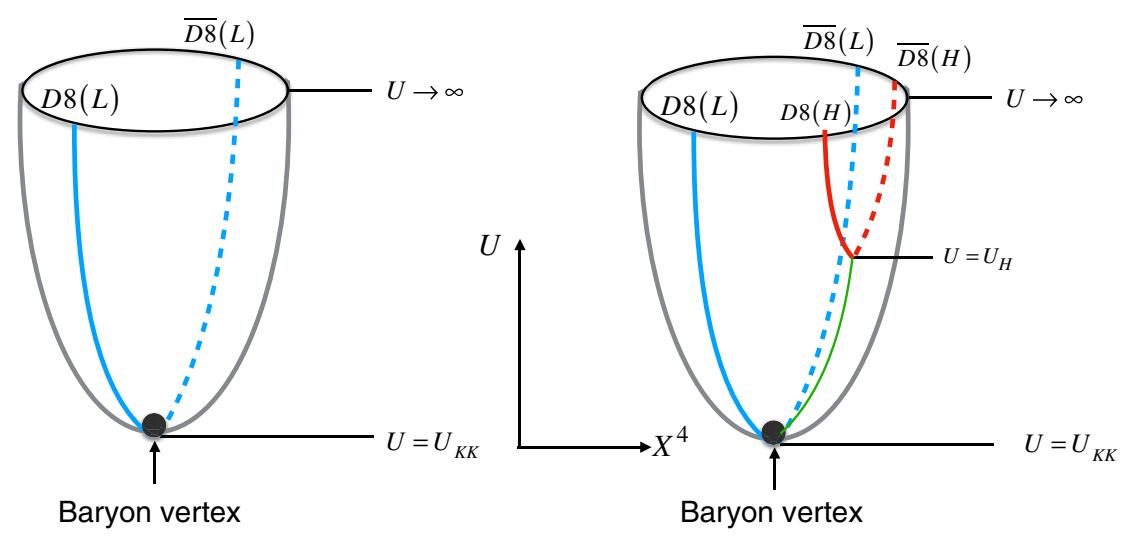

FIG. 1. The various D-brane configurations in the WSS model. Left: The configuration of the standard WSS model according to Table I. The bulk geometry is produced by $N_{c}$ coincident D4-branes which represent "colors" in QCD. The flavors are introduced into the model by embedding $N_{f}$ pairs of coincident D8/D8-branes at the antipodal position of the bulk geometry. $U$ refers to the holographic direction and $X^{4}$ is compactified on $S^{1}$. The D4'-brane as the baryon vertex looks like a point in the $U-X^{4}$ plane. Mesons are created by the open string on the flavored D8/D8 -branes while baryons are created by the wrapped D4'-branes. Right: The WSS model with heavy flavor. An additional pair of flavored D8 $/ \overline{\mathrm{D} 8}$-brane (denoted by the red line) as the heavy-flavored (H) brane separated from the other $N_{f}$ pairs of light-flavored (L) D8/D8-branes with a HL string (denoted by the green line) is embedded. The baryon vertex contains heavy flavor in this configuration through the HL string.

the nonzero vacuum expectation value (VEV) of the HL string. Therefore, we could interpret the multiplets created by the HL string as the heavy-flavored mesons with massive flavored (heavy-flavored) quarks. Actually, this mechanism to acquire mass is nothing but the "Higgs mechanism" in string theory. So let us replace the gauge fields on the light flavor branes by its matrix-valued form to involve the heavy flavor,

$$
\mathcal{A}_{a} \rightarrow \mathcal{A}_{a}=\left(\begin{array}{cc}
\mathcal{A}_{a} & \Phi_{a} \\
-\Phi_{a}^{\dagger} & 0
\end{array}\right)
$$

In our notation, $\mathcal{A}_{a}$ is an $N_{f} \times N_{f}$ matrix-valued 1-form while $\mathcal{A}_{a}$ is an $\left(N_{f}+1\right) \times\left(N_{f}+1\right)$ matrix-valued 1 -form. $\Phi_{a}$ is an $N_{f} \times 1$ matrix-valued vector which represents HL meson field and the index runs over the light flavor brane. Thus, the field strength of $\mathcal{A}_{a}$ also becomes matrix-valued as a 2 -form,

$\mathcal{F}_{a b} \rightarrow \mathcal{F}_{a b}=\left(\begin{array}{cc}\mathcal{F}_{a b}-\Phi_{[a} \Phi_{b]}^{\dagger} & \partial_{[a} \Phi_{b]}+\mathcal{A}_{[a} \Phi_{b]} \\ -\partial_{[a} \Phi_{b]}^{\dagger}-\Phi_{[a}^{\dagger} \mathcal{A}_{b]} & -\Phi_{[a}^{\dagger} \Phi_{b]}\end{array}\right)$,

where $\mathcal{F}_{a b}$ refers to the field strength of $\mathcal{A}_{a}$. Imposing (2.1) (2.2) into D8-brane action (C1) and keep the quadric terms of $\Phi_{a}$, it leads to a Yang-Mills (YM) action ${ }^{3}$

\footnotetext{
${ }^{3}$ We do not given the explicit formula of the CS term with HL fields since it is independent on the metric; thus, it is irrelevant to the glueball-baryon interaction.
}

$$
\begin{aligned}
S_{\mathrm{DBI}}^{\mathrm{YM}}= & -\frac{1}{4}\left(2 \pi \alpha^{\prime}\right)^{2} T_{8} \operatorname{Tr} \int_{\mathrm{D} 8 / \overline{\mathrm{D} 8}} d^{9} x e^{-\Phi} \sqrt{-g} g^{a b} g^{c d} \mathcal{F}_{a c} \mathcal{F}_{b d} \\
= & -\frac{1}{4}\left(2 \pi \alpha^{\prime}\right)^{2} T_{8} \int_{\mathrm{D} 8 / \overline{\mathrm{D} 8}} d^{9} x e^{-\Phi} \\
& \times \sqrt{-g}\left[g^{a b} g^{c d} \operatorname{Tr}\left(\mathcal{F}_{a c} \mathcal{F}_{b d}-\alpha_{a c} \mathcal{F}_{b d}-\mathcal{F}_{a c} \alpha_{b d}\right)\right. \\
& \left.-2 g^{a b} g^{c d} f_{a c}^{\dagger} f_{b d}\right],
\end{aligned}
$$

where

$$
\begin{aligned}
& f_{a b}=\partial_{[a} \Phi_{b]}+A_{[a} \Phi_{b]}, \\
& f_{a b}^{\dagger}=-\partial_{[a} \Phi_{b]}^{\dagger}-\Phi_{[a}^{\dagger} A_{b]}, \quad \alpha_{a b}=\Phi_{[a} \Phi_{b]}^{\dagger} .
\end{aligned}
$$

We should notice that from the full formula of the DBI action, it would contain an additional term of the transverse mode $\Psi$ of D8/D8-branes as shown in Appendix B. And this term could be written as

$$
\begin{aligned}
S_{\Psi}^{\mathrm{D} 8}= & -\tilde{T}_{8} \operatorname{Tr} \int_{\mathrm{D} 8 / \overline{\mathrm{D} 8}} d^{9} x e^{-\Phi} \sqrt{-\operatorname{det} g} \\
& \times\left\{\frac{1}{2} D_{a} \Psi D^{a} \Psi+\frac{1}{4}[\Psi, \Psi]^{2}\right\},
\end{aligned}
$$

with $D_{a} \Psi=\partial_{a} \Psi+\left[\mathbf{A}_{a}, \Psi\right]$ and $\tilde{T}_{8}=\left(2 \pi \alpha^{\prime}\right)^{2} T_{8}$. In the case of $N_{f}$ pairs of light-flavored D8/D8 branes separated from one pair of heavy-flavored $\mathrm{D} 8 / \overline{\mathrm{D} 8}$ branes, we can define the moduli solution of $\Psi$ with a finite VEV $v$ by the extrema of the potential contribution or $[\Psi,[\Psi, \Psi]]=0$ $[27,28]$ as 


$$
\Psi=\left(\begin{array}{cc}
-\frac{v}{N_{f}} \mathbf{1}_{N_{f}} & 0 \\
0 & v
\end{array}\right)
$$

So the action (2.5) could be rewritten by plugging the solution (2.6) into (2.5) as

$$
\begin{aligned}
S_{\Psi}= & -\tilde{T}_{8} v^{2} \frac{\left(N_{f}+1\right)^{2}}{N_{f}^{2}} \operatorname{Tr} \int d^{4} x \\
& \times \int_{-\infty}^{+\infty} d Z e^{-\Phi} \sqrt{-\operatorname{det} g} g^{a b} \Phi_{a}^{\dagger} \Phi_{b},
\end{aligned}
$$

which is exactly the mass term of the HL field. Then perform the rescaling (C3), we could obtain the classical equations of motions for $\Phi_{a}$ from (2.3) (2.7) as

$$
\begin{aligned}
& D_{M} D_{M} \Phi_{N}-D_{N} D_{M} \Phi_{M}+2 \mathcal{F}_{N M} \Phi_{M}+\mathcal{O}\left(\lambda^{-1}\right)=0 . \\
& D_{M}\left(D_{0} \Phi_{M}-D_{M} \Phi_{0}\right)-\mathcal{F}^{0 M} \Phi_{M} \\
& \quad-\frac{1}{64 \pi^{2} a} \epsilon_{M N P Q} K_{M N P Q}+\mathcal{O}\left(\lambda^{-1}\right)=0
\end{aligned}
$$

where $x^{M}=\left\{x^{i}, Z\right\}, i=1,2,3$ and the 4-form $K_{M N P Q}$ is given as

$$
\begin{aligned}
K_{M N P Q}= & \partial_{M} \mathcal{A}_{N} \partial_{P} \Phi_{Q}+\mathcal{A}_{M} \mathcal{A}_{N} \partial_{P} \Phi_{Q} \\
& +\partial_{M} \mathcal{A}_{N} \mathcal{A}_{P} \Phi_{Q}+\frac{5}{6} \mathcal{A}_{M} \mathcal{A}_{N} \mathcal{A}_{P} \Phi_{Q}
\end{aligned}
$$

Since the holographic approach is valid in the strong coupling limit $\lambda \rightarrow \infty$, the contributions from $\mathcal{O}\left(\lambda^{-1}\right)$ have been dropped off. Note that the light flavored gauge field $\mathcal{A}_{a}$ satisfies the equations of motion obtained by varying the action $(\mathrm{C} 1)$, so their solution remains to be $(\mathrm{C} 2)$ in the large $\lambda$ limit. And we could further define $\Phi_{a}=\phi_{a} e^{ \pm i m_{H} x^{0}}$ in the heavy quark limit i.e., $m_{H} \rightarrow \infty$ as in $[25,26]$ so that $D_{0} \Phi_{M}=\left(D_{0} \pm i m_{H}\right) \phi_{M}$ where " \pm " corresponds to quark and antiquark, respectively. By keeping these in mind, altogether we find the full solution for (2.8) as

$$
\begin{aligned}
\phi_{0} & =-\frac{1}{1024 a \pi^{2}}\left[\frac{25 \rho}{2\left(x^{2}+\rho^{2}\right)^{5 / 2}}+\frac{7}{\rho\left(x^{2}+\rho^{2}\right)^{3 / 2}}\right] \chi, \\
\phi_{M} & =\frac{\rho}{\left(x^{2}+\rho^{2}\right)^{3 / 2}} \sigma_{M} \chi
\end{aligned}
$$

where $\chi$ is a spinor independent on $x^{M}$. Then in the double limit i.e., $\lambda \rightarrow \infty$ followed by $m_{H} \rightarrow \infty$, the Hamiltonian for the collective modes involving the heavy flavor could be calculated as in (C7) by following the procedures in Appendix C.

\section{GLUEBALL-BARYON INTERACTION WITH HEAVY FLAVOR}

The dynamic of the free glueball is reviewed in Appendix A, so in this section we will focus on the interaction of the glueball and baryon with heavy flavor characterized by the collective Hamiltonian. As the glueball field is included by the metric fluctuations, the ChernSimons (CS) term is independent on the metric; thus, it does not involve the glueball-baryon interaction. Hence, let us start with the five-dimensional YM action plus the mass term which are collected in (2.3) (2.7). The onshell form of (2.3) (2.7) corresponds to the effective interaction Hamiltonian of the glueball and heavy-flavored baryon through the relation $S^{\text {onshel }}=-\int d t H_{G-B}$, accordingly we first need to solve the eigenvalue equations for function $H_{E, D, T}$ in large $\lambda$ limit.

The eigenvalue equations for $H_{E, D, T}$ are given in (A9) and (A13). In the rescaled coordinate $Z \rightarrow \lambda^{-1 / 2} Z$, the equations are written as

$$
\begin{aligned}
& H_{E}^{\prime \prime}(Z)+\left(\frac{1}{Z}+\frac{2 Z}{\lambda}\right) H_{E}^{\prime}(Z)+\left(\frac{16}{3 \lambda}+\frac{M_{E}^{2}}{M_{K K}^{2}} \frac{1}{\lambda}\right) H_{D}(Z) \\
& \quad+\mathcal{O}\left(\lambda^{-2}\right)=0,
\end{aligned}
$$

$$
\begin{aligned}
& H_{D, T}^{\prime \prime}(Z)+\left(\frac{1}{Z}+\frac{2 Z}{\lambda}\right) H_{D, T}^{\prime}(Z)+\frac{M_{D}^{2}}{M_{K K}^{2}} \frac{1}{\lambda} H_{D, T}(Z) \\
& \quad+\mathcal{O}\left(\lambda^{-2}\right)=0,
\end{aligned}
$$

and they could be easily solved as

$$
\begin{aligned}
H_{E}(z)= & \mathcal{C}_{E}\left(1-\frac{3 M_{E}^{2}+16 M_{K K}^{2}}{12 M_{K K}^{2} \lambda} Z^{2}\right) \lambda^{-1 / 2} N_{c}^{-1} M_{K K}^{-1} \\
& +\mathcal{O}\left(\lambda^{-3 / 2}\right), \\
H_{D, T}(z)= & \mathcal{C}_{D, T}\left(1-\frac{M_{D, T}^{2}}{4 M_{K K}^{2} \lambda} Z^{2}\right) \lambda^{-1 / 2} N_{c}^{-1} M_{K K}^{-1} \\
& +\mathcal{O}\left(\lambda^{-3 / 2}\right) .
\end{aligned}
$$

Next we perform the rescaling as in (C3), then insert the BPST solution (C2) for the gauge field $\mathcal{A}$ and (A9) for the heavy-light meson field $\Phi_{a}$ into the action (2.3) (2.7). Afterwards by plugging the metric (A6) plus the dilaton (A7) with the solution (3.2) and various fluctuations which include the exotic scalar, dilatonic scalar and tensor glueball field all given in Appendix A into the action (2.3) (2.7), the onshell form of action (2.3) (2.7) could be obtained with the dimensionless variable $x^{\mu} \rightarrow x^{\mu} / M_{K K}, \mathcal{A}_{\mu} \rightarrow$ $\mathcal{A}_{\mu} M_{K K}$ as

$$
\begin{aligned}
S_{G_{E, D, T}-B}^{\text {onshell }}= & a \mathcal{C}_{E, D, T} \int d^{4} x d \operatorname{Tr} \operatorname{Tr}\left[\lambda^{1 / 2} \mathcal{L}_{1 / 2}^{E, D, T}+\mathcal{L}_{0}^{E, D, T}\right. \\
& \left.+\lambda^{-1 / 2} \mathcal{L}_{-1 / 2}^{E, D}+\lambda^{-1 / 2} \mathcal{L}_{\Psi}^{E, D, T}+\mathcal{O}\left(\lambda^{-1} m_{H}^{0}\right)\right]
\end{aligned}
$$


where $a=\frac{1}{216 \pi^{3}}$, " $E, D, T$ " refers, respectively, to "exotic scalar, dilatonic scalar, and tensor glueball." Although the above calculation is very straightforward, the explicit forms of $\mathcal{L}_{1 / 2,0,-1 / 2}^{E, D, T}$ and $\mathcal{L}_{\Psi}^{E, D, T}$ are quite lengthy. So we summarize the full formulas of $\mathcal{L}_{1 / 2,0,-1 / 2}^{E, D, T}$ and $\mathcal{L}_{\Psi}^{E, D, T}$ with some essential instructions in Appendix D and here skip to the final results. Using the relation $S_{G-B}^{\text {onshell }}=-\int d t H_{G-B}\left(t, \mathcal{X}^{s}\right)$, the dimensionless interaction Hamiltonians are computed as, $^{4}$

$$
\begin{aligned}
H_{G_{E}-B}\left(t, \mathcal{X}^{s}\right)= & -\mathcal{C}_{E} \lambda^{-1 / 2} M_{K K}^{-1}\left(5 m_{H}^{2} \pi^{2} a+\frac{15 m_{H}}{32 \rho^{2}}\right) G_{E} \chi^{\dagger} \chi \\
& +\mathcal{O}\left(\lambda^{-1} m_{H}^{0}\right) \\
H_{G_{D}-B}\left(t, \mathcal{X}^{s}\right)= & \mathcal{C}_{D} \lambda^{-1 / 2} M_{K K}^{-1}\left(\frac{3 m_{H}}{8 \rho^{2}}-6 m_{H}^{2} \pi^{2} a\right) G_{D} \chi^{\dagger} \chi \\
& +\mathcal{O}\left(\lambda^{-1} m_{H}^{0}\right) \\
H_{G_{T}-B}\left(t, \mathcal{X}^{s}\right)= & -\mathcal{C}_{T} \lambda^{-1 / 2} M_{K K}^{-1}\left(\frac{7}{2} m_{H}^{2} \pi^{2} a+\frac{m_{H}}{4 \rho^{2}}\right) G_{T} \chi^{\dagger} \chi \\
& +\mathcal{O}\left(\lambda^{-1} m_{H}^{0}\right),
\end{aligned}
$$

The constants $\mathcal{C}_{E, D, T}$ are determined by the eigenvalue equations for $H_{E, D, T}$, and they depend on the mass of the various glueballs. We numerically evaluate $\mathcal{C}_{E, D, T}$ in Table II with the corresponding glueball mass. Notice that the operator $G_{E, D, T}$ satisfies the equations of motion by varying action (A10) (A14); thus, its classical solution is $G_{E, D, T}=\frac{1}{2}\left(e^{-i M_{E, D, T} t}+\right.$ c.c $)$ and it is time dependent. On the other hand, the spinor $\chi$ has to be quantized by its anticommutation relation $\left\{\chi_{\alpha}, \chi_{\beta}^{\dagger}\right\}=\delta_{\alpha \beta}$ in the full quantum field theory so $\chi^{\dagger} \chi$ is the number operator of heavy quarks. Therefore, in our theory, the glueball field could be treated as the classical field while baryon is quantized in the moduli space and we can identify $\chi^{\dagger} \chi=N_{Q}$ as the number of heavy quarks in a baryonic bound state. Moreover, the Hamiltonians in (3.4) is definitely suitable to be perturbations to the quantum mechanics (C7) since they are all proportional to $\lambda^{-1 / 2}$ in the large $\lambda$ limit. Then the interaction of the glueball and heavy-flavored baryon could be accordingly described by using the method of time-dependent perturbation in the quantum mechanical system. Last but not least, the decay rates/width $\Gamma$ can be evaluated by using the standard technique for the timedependent perturbation in quantum mechanics, which is given as

\footnotetext{
${ }^{4}$ The glueball field $G_{E, D, T}$ in (3.4) is dimensional which is in the unit of $M_{K K}$ while the other parameters are dimensionless.
}

TABLE II. The glueball mass spectrum $M_{E T}^{(n)}$ in the WSS model in the units of $M_{K K}$ is collected from [14] and the numerical values of the associated coefficients presented in (3.4) $\mathcal{C}_{E, D, T}$ are evaluated.

\begin{tabular}{llllll}
\hline \hline Excitation of glueball $(n)$ & $n=0$ & $n=1$ & $n=2$ & $n=3$ & $n=4$ \\
\hline Glueball mass $M_{E}^{(n)}$ & 0.901 & 2.285 & 3.240 & 4.149 & 5.041 \\
Glueball mass $M_{D, T}^{(n)}$ & 1.567 & 2.485 & 3.373 & 4.252 & 5.124 \\
\hline \hline
\end{tabular}

\begin{tabular}{lrrrrr}
\hline \hline The coefficients & $n=0$ & $n=1$ & $n=2$ & $n=3$ & $n=4$ \\
\hline $\mathcal{C}_{E}$ & 144.545 & 114.871 & 131.283 & 146.259 & 157.832 \\
$\mathcal{C}_{D}$ & 29.772 & 36.583 & 42.237 & 47.220 & 51.724 \\
$\mathcal{C}_{T}$ & 72.927 & 89.609 & 103.46 & 115.664 & 126.696 \\
\hline \hline
\end{tabular}

$$
\begin{aligned}
\frac{\Gamma_{B \rightarrow G+X}}{m_{H}} & =\frac{1}{m_{H}}\left|\int d t\left\langle i\left|H_{G-B}\left(t, \mathcal{X}^{s}\right)\right| j\right\rangle e^{-i\left(E_{i}-E_{j}\right) t}\right|^{2}, \\
& =\frac{1}{m_{H}}\left\langle i\left|H_{G-B}\left(\mathcal{X}^{s}\right)\right| j\right\rangle^{2} \delta\left(E_{j}-E_{i}-M_{E, D, T}\right),
\end{aligned}
$$

$|i\rangle,|j\rangle, E_{i, j}$ refers to the eigenstate and the associated eigenvalue of (C7). And the above decay occurs only if several physical quantities e.g., energy, total angular momentum $J$, are also conserved. Note that the interaction Hamiltonians in (3.4) are independent on $Z$, so $\left\langle i\left|H_{G-B}\left(\mathcal{X}^{s}\right)\right| j\right\rangle$ would be vanished unless the states $|i\rangle$, $|j\rangle$ take the same quantum number of $n_{Z}$ and $l$. The Hamiltonians in (3.4) can also describe the decay of an antibaryon if we replace $m_{H}$ by $-m_{H}$.

With the perturbed Hamiltonian in (3.4), this model includes various decays of heavy-flavored hadrons involving the glueball. So we are going to examine the possible transitions involving one glueball with the leading lowenergy excited baryon states $n_{\rho} \leq 5$. Since our concern is the situation of two-flavored meson, we could follow [22] by setting $\frac{l}{2}=J=0, N_{Q}=1, N_{c}=3$ in order to fit the experimental data of the (pseudo) scalar meson states with one heavy flavor. Then let us first take account into the energy conservation $E\left(n_{\rho}=n_{\rho}^{\prime}+\Delta n_{\rho}, l=0, N_{B}=1, n_{Z}\right)-$ $E\left(n_{\rho}^{\prime}, l=0, N_{B}=1, n_{Z}\right) \equiv \mathcal{E}\left(\Delta n_{\rho}\right)=M_{E, D, T}^{(n)}$ if the transition of hadron decay would happen, where $M_{E, D, T}^{(n)}$ refers to the glueball mass given in Table II and $E\left(n_{\rho}, l, N_{B}, n_{Z}\right)$ refers to the baryonic spectrum in (C9). By keeping these in mind, the following relations are picked out,

$$
\begin{aligned}
& \mathcal{E}\left(\Delta n_{\rho}=3\right) / M_{D, T}^{(n=1)} \simeq 0.986 \\
& \mathcal{E}\left(\Delta n_{\rho}=4\right) / M_{E}^{(n=2)} \simeq 1.008
\end{aligned}
$$

while $\mathcal{E}\left(\Delta n_{\rho}\right), n_{\rho} \leq 5$ with $\Delta n_{\rho}=0,1,2$ does not match to any $M_{E, D, T}^{(n)}$. Hence we could find the following possible decays involving the glueball according to (3.6), 
I: Baryonic $\left|J=0, n_{\rho}=3\right\rangle$

$$
\rightarrow\left|G_{D}^{(n=1)}, J^{P C}=0^{++}\right\rangle+\text {Baryonic }\left|J=0, n_{\rho}=0\right\rangle
$$

II: Baryonic $\left|J=0, n_{\rho}=4\right\rangle$

$$
\rightarrow\left|G_{D}^{(n=1)}, J^{P C}=0^{++}\right\rangle+\text {Baryonic }\left|J=0, n_{\rho}=1\right\rangle
$$

III: Baryonic $\left|J=0, n_{\rho}=5\right\rangle$

$$
\rightarrow\left|G_{D}^{(n=1)}, J^{P C}=0^{++}\right\rangle+\text {Baryonic }\left|J=0, n_{\rho}=2\right\rangle
$$

IV: Baryonic $\left|J=0, n_{\rho}=3\right\rangle$

$$
\rightarrow\left|G_{T}^{(n=1)}, J^{P C}=2^{++}\right\rangle+\text {Baryonic }\left|J=0, n_{\rho}=0\right\rangle
$$

$\mathrm{V}:$ Baryonic $\left|J=0, n_{\rho}=4\right\rangle$

$$
\rightarrow\left|G_{T}^{(n=1)}, J^{P C}=2^{++}\right\rangle+\text {Baryonic }\left|J=0, n_{\rho}=1\right\rangle
$$

VI: Baryonic $\left|J=0, n_{\rho}=5\right\rangle$

$$
\rightarrow\left|G_{T}^{(n=1)}, J^{P C}=2^{++}\right\rangle+\text {Baryonic }\left|J=0, n_{\rho}=2\right\rangle
$$

VII: Baryonic $\left|J=0, n_{\rho}=4\right\rangle$

$$
\rightarrow\left|G_{E}^{(n=2)}, J^{P C}=0^{++}\right\rangle+\text {Baryonic }\left|J=0, n_{\rho}=0\right\rangle
$$

VIII: Baryonic $\left|J=0, n_{\rho}=5\right\rangle$

$$
\rightarrow\left|G_{E}^{(n=2)}, J^{P C}=0^{++}\right\rangle+\text {Baryonic }\left|J=0, n_{\rho}=1\right\rangle,
$$

where we have denoted the states by their quantum numbers and the associated decay rates $\Gamma$ are numerically evaluated in Table III by using the effective Hamiltonian in (3.4). Notice that the mass of the dilatonic and exotic scalar glueball in (3.7) are given as $M_{E}^{(n=2)} / M_{D}^{(n=1)} \simeq 1.30$ which is close to the mass ratio of the glueball candidates $f_{0}(1710)$ and $f_{0}(1500)$ as $M_{f_{0}(1710)} / M_{f_{0}(1500)} \simeq 1.14$, moreover all of them should be the state of $J^{P C}=0^{++}$. Accordingly, we could identify the dilatonic and exotic scalar glueball in (3.7) as $f_{0}(1500)$ and $f_{0}(1710)$, respectively, which are the two glueball candidates discussed frequently in many lectures.

If we furthermore consider the parity of baryonic states as discussed in [22], the above states with odd $n_{Z}$ in this model would correspond to the meson states with odd parity since the parity transformation is $Z \rightarrow-Z$. In this sense, the transition II, V, VII describes the decay of the heavy-flavored scalar (nonglueball) meson involving the

TABLE III. The corresponding decay rates in the units of $m_{H}$ to the transitions in (3.7) by setting $l=0, N_{Q}=1, N_{c}=3, N_{f}=2$.

\begin{tabular}{ccccc}
\hline \hline & I & II & III & IV \\
\hline$\Gamma$ & $0.0392 \lambda^{-1}$ & $0.0628 \lambda^{-1}$ & $0.0785 \lambda^{-1}$ & $0.1046 \lambda^{-1}$ \\
\hline \hline & $\mathrm{V}$ & $\mathrm{VI}$ & $\mathrm{VII}$ & $\mathrm{VIII}$ \\
\hline$\Gamma$ & $0.1674 \lambda^{-1}$ & $0.2093 \lambda^{-1}$ & $0.6316 \lambda^{-1}$ & $1.0527 \lambda^{-1}$ \\
\hline \hline
\end{tabular}

glueball, while the pure scalar meson with even parity is less evident according to the current experimental data. On the other hand, as the glueball states we discussed in this manuscript all have even parity, it implies that the parity of the transition I, III, IV, VI may be violated. We also notice that if $\frac{l}{2}=J$ is identified as the quantum number of the spin, the decay processes IV, V, VI in (3.7) involving the tensor glueball $J^{P C}=2^{++}$may be probably forbidden since the initial and final baryonic states are all pure scalars i.e., the total angular momentum may not be conserved in these transitions, ${ }^{5}$ and this result would be in agreement with the previous discussion in [19]. Therefore we could conclude that only the decay process VIII in (3.7) might be realistic. This transition describes the decay of the baryonic meson consisted of one heavy- and one light-flavored quark. So while the identification of the other transitions might be less clear, the transition VIII could be interpreted as the decay of the baryonic B-meson involving the glueball candidate $f_{0}(1710)$ as discussed e.g., in [8-10] since the corresponding quantum numbers of the states could be identified.

\section{SUMMARY}

In this paper, with the top-down approach of WSS model, we propose a holographic description of the decay of heavy-flavored meson involving the glueball. The HL field is introduced into the WSS model to describe the dynamics of heavy flavor, and it is created by the HL string with a pair of heavy-flavored D8 $/ \overline{\mathrm{D} 8}$-brane separated from the other light flavored D8/D8-brane. Since baryon in this model could be equivalently represented by the instanton configurations on the light-flavored brane and the glueball field is identified as the bulk gravitational waves, we solve the classical equations of motion for the HL field with instanton solution for the gauge fields. In the limitation of large $\lambda$ followed by large $m_{H}$, we derive the mass formula of the soliton as the onshell action of the flavor brane by taking account of the HL field and bulk gravitational waves. Then following the collectivization and quantization of the soliton in $[22,25,26]$, the effective Hamiltonian for the collective modes of heavy-flavored baryons is obtained which includes the interaction with the glueball. Afterwards, we examine the possible decay processes and compute the associated decay rates with the effective Hamiltonian. We find these decay rates are in agreement with the previous works by using this model as in [14-16,19] since they are proportional to $\lambda^{-1}$. Then by comparing the quantum numbers of the baryonic states with some experimental data and employing the identification of baryonic states in [22], we find that one decay process might be realistic and could be interpreted as the

\footnotetext{
${ }^{5}$ For a tensor glueball, we suggest considering a tensor field dependent on the coordinates of the moduli space $y_{I}$ in order to obtain the correct decay process. We would like to leave it for future study and focus on the scalar glueball in the current work.
} 
decay of baryonic B-meson involving the glueball candidate $f_{0}(1710)$ as discussed in [8-10]. Noteworthily according to lattice QCD $f_{0}(1710)$ is an excited state in the glueball candidates which is just consistent with that the glueball state discussed in transition VIII is also an excitation.

As an improvement of [19], this work provides an alternative way to investigate the interaction of the glueball and heavy-flavored baryons in the strong coupling system through the holographic approach of the underlying string theory. Although this approach is quite principal and contains few parameters, it is actually valid in the large $N_{c}$ limit. So phenomenological theories or models are always needed as a comparison with holography.

\section{ACKNOWLEDGMENTS}

I would like to thank Anton Rebhan, Josef Leutgeb, and Chao Wu for valuable comments and discussions. S. W. L. is supported by the research startup foundation of Dalian Maritime University in 2019.

\section{APPENDIX A: THE BULK SUPERGRAVITY AND GLUEBALL DYNAMICS IN THE WSS MODEL}

The WSS model is based on the $\mathrm{AdS}_{7} / \mathrm{CFT}_{6}$ correspondence of $N_{c}$ M5-branes in string theory which can be reduced to $N_{c}$ D4-branes compactified on $S^{1}$ in the tendimensional bulk. So taking the large $N_{c}$ limit, the bulk dynamic is described by the ten-dimensional type IIA supergravity action which is given as

$$
S_{\text {IIA }}=\frac{1}{2 k_{10}^{2}} \int d^{10} x \sqrt{-g} e^{-2 \Phi}\left(\mathcal{R}+4 \nabla_{M} \Phi \nabla^{M} \Phi-\frac{1}{2}\left|F_{4}\right|^{2}\right),
$$

where $\Phi$ denotes the dilaton field, $2 k_{10}^{2}=16 G_{10} / g_{s}^{2}=$ $(2 \pi)^{7} l_{s}^{8}$. $\mathcal{R}, G_{10}$ is the ten-dimensional scalar curvature and Newton constant, respectively. $F_{4}=d C_{3}$ is the field strength of the Romand-Romand (R-R) 3-form $C_{3}$. The geometrical solution for the bulk metric is given as

$$
\begin{gathered}
d s^{2}=\left(\frac{U}{R}\right)^{3 / 2}\left[\eta_{\mu \nu} d X^{\mu} d X^{\nu}+f(U)\left(d X^{4}\right)^{2}\right] \\
+\left(\frac{R}{U}\right)^{3 / 2}\left[\frac{d U^{2}}{f(U)}+U^{2} d \Omega_{4}^{2}\right] \\
f(U)=1-\frac{U_{K K}^{3}}{U^{3}}, \quad e^{\Phi}=\left(\frac{U}{R}\right)^{3 / 4} \\
F_{4}=\frac{2 \pi N_{c}}{V_{4}} \epsilon_{4}, \quad R^{3}=\pi g_{s} N_{c} l_{s}^{3}
\end{gathered}
$$

with a periodic condition for $X^{4}$,

$$
X^{4} \sim X^{4}+2 \pi \delta X^{4}, \quad \delta X^{4}=\frac{1}{M_{K K}} .
$$

And the $r, z, Z$ coordinate used in the paper is defined as

$$
\begin{aligned}
U^{3} & =U_{K K}^{3}+U_{K K} z^{2}, \quad Z=\frac{z}{U_{K K}}, \\
1+Z^{2} & =\frac{r^{6}}{r_{K K}^{6}}, \quad U_{K K}=\frac{r_{K K}^{2}}{4 R} .
\end{aligned}
$$

Note that $\epsilon_{4}$ represents a unit volume element on $S^{4} . g_{s}, l_{s}$ denotes the string coupling constant and the length of string. The indices $\mu, \nu$ in (A2) run from 0 to 3 . Additionally we could define the QCD variables in terms of

$$
\lambda=g_{\mathrm{YM}}^{2} N_{c}, \quad g_{\mathrm{YM}}^{2}=2 \pi g_{s} l_{s} M_{K K},
$$

where $g_{\mathrm{YM}}, \lambda$ respectively denotes the Yang-Mills and the 't Hooft coupling constant.

In this model, the glueball fields are identified as the gravitational fluctuations to the bulk solution (A2); thus, we could rewrite the metric as $G_{M N} \rightarrow G_{M N}^{(0)}+\delta G_{M N}$ in order to involve the glueball field. The ten-dimensional metric reduced from eleven-dimensional supergravity with gravitational fluctuations is

$g_{\mu \nu}=\frac{r^{3}}{L^{3}}\left[\left(1+\frac{L^{2}}{2 r^{2}} \delta G_{11,11}\right) \eta_{\mu \nu}+\frac{L^{2}}{r^{2}} \delta G_{\mu \nu}\right]$,

$g_{44}=\frac{r^{3} f}{L^{3}}\left[1+\frac{L^{2}}{2 r^{2}} \delta G_{11,11}+\frac{L^{2}}{r^{2} f} \delta G_{44}\right]$,

$g_{r r}=\frac{L}{r f}\left(1+\frac{L^{2}}{2 r^{2}} \delta G_{11,11}+\frac{r^{2} f}{L^{2}} \delta G_{r r}\right)$,

$g_{r \mu}=\frac{r}{L} \delta G_{r \mu}, \quad g_{\Omega \Omega}=\frac{r}{L}\left(\frac{L}{2}\right)^{2}\left(1+\frac{L^{2}}{2 r^{2}} \delta G_{11,11}\right)$,

with the dilaton

$$
e^{4 \Phi / 3}=\frac{r^{2}}{L^{2}}\left(1+\frac{L^{2}}{r^{2}} \delta G_{11,11}\right) .
$$

Since different formulas of $\delta G_{M N}$ corresponds to various glueball, in this paper we consider the following forms of $\delta G_{M N}$ :

\section{The exotic scalar glueball}

The exotic scalar glueball corresponds to the exotic polarizations of the bulk graviton whose quantum number is $J^{C P}=0^{++}$. The eleven-dimensional components of $\delta G_{M N}$ are given as 


$$
\begin{aligned}
\delta G_{44} & =-\frac{r^{2}}{L^{2}} f(r) H_{E}(r) G_{E}(x), \\
\delta G_{\mu \nu} & =\frac{r^{2}}{L^{2}} H_{E}(r)\left[\frac{1}{4} \eta_{\mu \nu}-\left(\frac{1}{4}+\frac{3 r_{K K}^{6}}{5 r^{6}-2 r_{K K}^{6}}\right) \frac{\partial_{\mu} \partial_{\nu}}{M_{E}^{2}}\right] G_{E}(x), \\
\delta G_{11,11} & =\frac{r^{2}}{4 L^{2}} H_{E}(r) G_{E}(x), \\
\delta G_{r r} & =-\frac{L^{2}}{r^{2}} \frac{1}{f(r)} \frac{3 r_{K K}^{6}}{5 r^{6}-2 r_{K K}^{6}} H_{E}(r) G_{E}(x), \\
\delta G_{r \mu} & =\frac{90 r^{7} r_{K K}^{6}}{M_{E}^{2} L^{2}\left(5 r^{6}-2 r_{K K}^{6}\right)^{2}} H_{E}(r) \partial_{\mu} G_{E}(x),
\end{aligned}
$$

with the eigenvalue equation for function $H_{E}(r)$ as

$$
\begin{aligned}
\frac{1}{r^{3}} & \frac{d}{d r}\left[r\left(r^{6}-r_{K K}^{6}\right) \frac{d}{d r} H_{E}(r)\right] \\
& +\left[\frac{432 r^{2} r_{K K}^{12}}{\left(5 r^{6}-2 r_{K K}^{6}\right)^{2}}+L^{4} M_{E}^{2}\right] H_{E}(r)=0 .
\end{aligned}
$$

In the ten-dimensional bulk, the above components in (A8) satisfy the asymptotics $\delta G_{44}=-4 \delta G_{11}=-4 \delta G_{22}=$ $-4 \delta G_{33}=-4 \delta G_{11,11}$ for $r \rightarrow \infty$. Plugging the solution (A2) and the fluctuations (A8) with the eigenvalue equation (A9) into the action (A1), it leads to the kinetic term of the exotic scalar glueball,

$$
S_{G_{E}(x)}=-\frac{1}{2} \int d^{4} x\left[\left(\partial_{\mu} G_{E}\right)^{2}+M_{E}^{2} G_{E}^{2}\right],
$$

where the pre-factor in (A10) has been normalized to $-1 / 2$ by choosing the boundary value of $H_{E}(r)$.

\section{The dilatonic and tensor glueball}

The fluctuations of the metric,

$$
\begin{aligned}
\delta G_{11,11} & =-3 \frac{r^{2}}{L^{2}} H_{D}(r) G_{D}(x), \\
\delta G_{\mu \nu} & =\frac{r^{2}}{L^{2}} H_{D}(r)\left[\eta^{\mu \nu}-\frac{\partial^{\mu} \partial^{\nu}}{M_{D}^{2}}\right] G_{D}(x),
\end{aligned}
$$

corresponds to another mode of the scalar glueball $0^{++}$. We employ "dilatonic" for the upon mode since $\delta G_{11,11}$ reduces to the ten-dimensional dilaton.

Besides the tensor glueball corresponds to the metric fluctuations with a transverse traceless polarization whose quantum number is $J^{C P}=2^{++}$. We can choose the following components of the graviton polarizations as tensor glueball field,

$$
\delta G_{\mu \nu}=-\frac{r^{2}}{L^{2}} H_{T}(r) T_{\mu \nu}(x),
$$

where $T_{\mu \nu} \equiv \mathcal{T}_{\mu \nu} G_{T}(x) . \mathcal{T}_{\mu \nu}$ is a constant symmetric tensor satisfying the normalization and traceless condition $\mathcal{T}_{\mu \nu} \mathcal{T}^{\mu \nu}=1, \eta^{\mu \nu} \mathcal{T}_{\mu \nu}=0$. The functions $H_{D, T}(r)$ satisfies the eigenvalue equation,

$\frac{1}{r^{3}} \frac{d}{d r}\left[r\left(r^{6}-r_{K K}^{6}\right) \frac{d}{d r} H_{D, T}(r)\right]+L^{4} M_{D, T}^{2} H_{D, T}(r)=0$.

We can also obtain the kinetic action of the dilatonic scalar and tensor glueball as

$$
\begin{aligned}
S_{G_{D}(x)} & =-\frac{1}{2} \int d^{4} x\left[\left(\partial_{\mu} G_{D}\right)^{2}+M_{D}^{2} G_{D}^{2}\right], \\
S_{T(x)} & =-\frac{1}{4} \int d^{4} x\left[T_{\mu \nu}\left(\partial^{2}-M_{T}^{2}\right) T^{\mu \nu}\right],
\end{aligned}
$$

once the solution (A2) and fluctuations (A11) (A12) with eigenvalue equation (A13) are imposed on the action (A1) and the boundary value of $H_{D, T}$ has to been determined by the normalization conditions in (A14).

\section{APPENDIX B: THE FULL Dp-BRANE ACTION AND THE EMBEDDING OF THE PROBE BRANES}

\section{The complete DBI action}

We give the complete formula of the Dp-brane here and it could also be reviewed in many textbooks of string theory, Let us consider $D$ dimensional spacetime parametrized by $\left\{X^{\mu}\right\}, \mu=0,1 \ldots D-1$ with a stack of Dp-branes. In this subsection, the indices $a, b=0,1 \ldots p$ and $i, j, k=p+$ $1 \ldots D-1$ denote respectively the directions parallel and vertical to the Dp-branes. The complete bosonic action of a Dp-branes is

$$
S_{\mathrm{D}_{p}-\text { branes }}=S_{\mathrm{DBI}}+S_{\mathrm{CS}}
$$

where [27]

$$
\begin{aligned}
S_{\mathrm{DBI}} & \left.=-T_{p} \mathrm{~S} \operatorname{Tr} \int d^{p+1} \xi e^{-\Phi} \sqrt{-\operatorname{det}\left\{\left[E_{a b}+E_{a i}\left(Q^{-1}-\delta\right)^{i j} E_{j b}+2 \pi \alpha^{\prime} F_{a b}\right] Q^{i}\right.}{ }_{j}\right\} \\
S_{\mathrm{CS}} & =\mu_{p} \sum_{n=0,1} \int_{\mathrm{D}_{p}-\text { branes }} C_{p-2 n+1} \wedge \frac{\left(B+2 \pi \alpha^{\prime} F\right)^{n}}{n !}, \\
Q_{j}^{i} & =\delta^{i} j+2 \pi \alpha^{\prime}\left[\varphi^{i}, \varphi^{k}\right] E_{k j}, \quad E_{\mu \nu}=g_{\mu \nu}+B_{\mu \nu} .
\end{aligned}
$$


We have denoted the metric of the $D$ dimensional spacetime and the 2 -form field as $g_{\mu \nu}, B_{\mu \nu}$, respectively. $F$ is the gauge field strength defined on the D-brane and "STr" refers to the "symmetric trace." We use $\varphi^{i}$ 's to represent the transverse modes of the Dp-branes which are given by the T-duality relation $2 \pi \alpha^{\prime} \varphi^{i}=X^{i}$. So the DBI action in (B2) could be expanded as

$$
\begin{aligned}
S_{\mathrm{BDI}}= & -T_{p} \operatorname{Tr} \int d^{p+1} \xi e^{-\Phi} \sqrt{-g}\left[1+\frac{1}{4}\left(2 \pi \alpha^{\prime}\right)^{2} F_{a b} F^{a b}\right. \\
& \left.+\frac{1}{2} D_{a} \varphi^{i} D_{a} \varphi^{i}+\frac{1}{4}\left[\varphi^{i}, \varphi^{j}\right]^{2}\right]+ \text { high orders. }
\end{aligned}
$$

The 2-form field $B$ has been gauged away. The gauge field $A_{a}$ and scalar field $\varphi^{i}$ 's are all in the adjoint representation of $U(N)$. Note that there is only one transverse coordinate

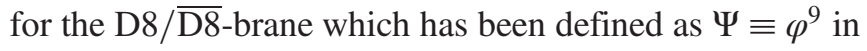
the main text.

\section{Comments about the probe branes and strings}

Here let us briefly outline the embedding of the probe D8/ $\overline{\mathrm{D} 8}$-brane and the HL string. Using the bulk metric (A2), the induced metric on the probe $\mathrm{D} 8 / \overline{\mathrm{D} 8}$-branes is obtained as

$$
\begin{aligned}
d s_{\mathrm{D} 8 / \overline{\mathrm{D} 8}}^{2}= & \left(\frac{U}{R}\right)^{3 / 2}\left[f(U)+\left(\frac{R}{U}\right)^{3} \frac{U^{\prime 2}}{f(U)}\right]\left(d X^{4}\right)^{2} \\
& +\left(\frac{U}{R}\right)^{3 / 2} \eta_{\mu \nu} d X^{\mu} d X^{\nu}+\left(\frac{R}{U}\right)^{3 / 2} U^{2} d \Omega_{4}^{2},
\end{aligned}
$$

where $U^{\prime}=\frac{d U}{d X^{4}}$. Then insert the metric (B4) into the DBI action of $\mathrm{D} 8 / \overline{\mathrm{D} 8}$-branes, it yields the formula

$S_{\mathrm{D} 8 / \overline{\mathrm{D} 8}} \propto \int d^{4} x d U U^{4}\left[f(U)+\left(\frac{R}{U}\right)^{3} \frac{U^{\prime 2}}{f(U)}\right]^{1 / 2}$.

Hence we can obtain the equation of motion for the function $U\left(X^{4}\right)$ as

$$
\frac{d}{d X^{4}}\left(\frac{U^{4} f(U)}{\left[f(U)+\left(\frac{R}{U}\right)^{3} \frac{1}{f(U)} U^{\prime 2}\right]^{1 / 2}}\right)=0 .
$$

Using the boundary condition in [13], as $U\left(X^{4}=0\right)=U_{0}$ and $U^{\prime}\left(X^{4}=0\right)=0$, the generic solution for (B6) is computed as

$X^{4}(U)=E\left(U_{0}\right) \int_{U_{0}}^{U} d U \frac{(U)\left(\frac{R}{U}\right)^{3 / 2}}{f(U)\left[U^{8} f(U)-E^{2}\left(U_{0}\right)\right]^{1 / 2}}$,

where $E\left(U_{0}\right)=U_{0}^{4} f^{1 / 2}\left(U_{0}\right)$ and we have used $U_{0}$ to denotes the connected position of the $\mathrm{D} 8 / \overline{\mathrm{D} 8}$-branes.
Afterwards let us further introduce the coordinates $(r, \Theta)$ and $(y, z)$ which satisfy,

$$
\begin{aligned}
y & =r \cos \Theta, \quad z=r \sin \Theta, \\
U^{3} & =U_{K K}^{3}+U_{K K} r^{2}, \quad \Theta=\frac{2 \pi}{\beta} X^{4}=\frac{3}{2} \frac{U_{K K}^{1 / 2}}{R^{3 / 2}} .
\end{aligned}
$$

In the standard WSS model, the probe D8/D的-branes are embedded at $\Theta= \pm \frac{1}{2} \pi$ respectively i.e., the position of $y=0$, which exactly corresponds to the antipodal D8/ $\overline{\mathrm{D} 8}$ branes (blue) in Fig. 1. In this case, the solution for the embedding function is $X^{4}(U)=\frac{1}{4} \beta$ and $U_{0}=U_{K K}$. In addition, the (B7) also allows the nonantipodal solution if we choose $\Theta= \pm \Theta_{H} \neq \pm \frac{1}{2} \pi, \quad U_{0}=U_{H} \neq U_{K K}$ which corresponds to the nonantipodal D8/ $\overline{\mathrm{D} 8}$-branes (red) in Fig. 1. On the other hand, while each end point of the HL string could move along the flavored branes, in our setup it is stretched between the heavy- (nonantipodal) and light-

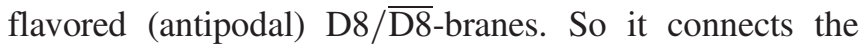
positions respectively on the heavy- and light-flavored D8 $/ \overline{\mathrm{D} 8}$-branes which are most close to each other and in the $U-X^{4}$ plane, they are the positions of $\left(U_{K K}, 0\right)$ on the light-flavored branes and $\left(U_{H}, 0\right)$ on the heavy-flavored branes. And this is the configuration of the HL string with minimal length i.e., the VEV.

\section{APPENDIX C: THE COLLECTIVE MODES OF THE BARYON AND ITS QUANTIZATION}

As the D4'-brane is identified as baryon in the WSS model, it is equivalent to the instanton configuration on the D8-branes according to the string theory. So the dynamic of the D8/ $\overline{\mathrm{D} 8}$-brane is given by the Dirac-Born-Infield (DBI) action plus the Chern-Simons (CS) action (B2) while the baryonic D4'-brane is identified as the instanton configuration of the gauge field strength on the $\mathrm{D} 8 / \overline{\mathrm{D} 8}$-brane. Altogether the action of the flavors with baryons can be simplified as a five-dimensional Yang-Mills (YM) plus CS action by integrating over the $S^{4}$ which is given as

$$
\begin{aligned}
S & =S_{\mathrm{YM}}+S_{\mathrm{CS}} . \\
S_{\mathrm{YM}} & =-\kappa \operatorname{Tr} \int d^{4} x d z e^{-\Phi} \sqrt{-g} g^{a b} g^{c d} \mathcal{F}_{a c} \mathcal{F}_{b d}, \\
S_{\mathrm{CS}} & =\frac{N_{c}}{24 \pi} \operatorname{Tr} \int d^{4} x d z\left(\mathcal{A} \mathcal{F}^{2}-\frac{1}{2} \mathcal{A}^{3} \mathcal{F}-\frac{1}{10} \mathcal{A}^{5}\right),
\end{aligned}
$$

where the indices $\alpha, \beta$ run over $X^{\mu}$ and $z$. In particular, in the situation of two flavors i.e., $N_{f}=2$, the classical instanton configuration could be adopted as the BelavinPolyakov-Schwarz-Tyupkin (BPST) solution which is given as 


$$
\begin{aligned}
& \mathcal{A}_{M}=-\bar{\sigma}_{M N} \frac{x^{N}}{x^{2}+\rho^{2}}, \quad M, N=1,2,3, z, \\
& \mathcal{A}_{0}=-\frac{i}{8 \pi^{2} a b^{3 / 2} x^{2}}\left[1-\frac{\rho^{4}}{\left(x^{2}+\rho^{2}\right)^{2}}\right]
\end{aligned}
$$

where $\mathcal{A}$ is $U(2)$ and $\mathcal{A}_{0}$ is $U(1)$ gauge field. The gauge field strength is defined as $\mathcal{F}=d \mathcal{A}+[\mathcal{A}, \mathcal{A}]{ }^{6}$ And $x^{2}=\left(x^{M}-X^{M}\right)^{2}, X^{M}$ 's are constants. Since the instanton size $\rho$ is of order $\lambda^{-1 / 2}$, it would be convenient to employ the rescaling,

$$
\left(x^{0}, x^{M}\right) \rightarrow\left(x^{0}, \lambda^{-1 / 2} x^{M}\right), \quad\left(\mathcal{A}_{0}, \mathcal{A}_{M}\right) \rightarrow\left(\mathcal{A}_{0}, \lambda^{1 / 2} \mathcal{A}_{M}\right),
$$

in order to obtain the explicit dependence of $\lambda$ in the actions in (C1). Inserting (C2) into the rescaled gauge field $\mathcal{A}$, the mass $M$ of the classical soliton could be evaluated by $S_{c l}^{\text {onshell }}=-\int d t M$. Afterwards the baryon states could be identified as Skyrmions so that the characteristics of baryon are reflected by their collective modes. Therefore we could quantize the classical soliton in the moduli space to obtain the baryon spectrum.

In the large $\lambda$ limit, the topology of the moduli space for $N_{f}=2$ case is given as $\mathbb{R}^{4} \times \mathbb{R}^{4} / \mathbb{Z}_{2}$ since the contribution of $\mathcal{O}\left(\lambda^{-1}\right)$ could be neglected. Then the collective coordinates $\left\{X^{M}\right\}$ parametrize the first $\mathbb{R}^{4}$ while the size $\rho$ and the $S U(2)$ orientation of the instanton parametrize $\mathbb{R}^{4} / \mathbb{Z}_{2}$. Let us denote the $S U(2)$ orientation as $a_{I}=\frac{y_{I}}{\rho}, I=1,2,3$, 4 with the normalization $\sum_{I=1}^{4} a_{I}^{2}=1$ so that the size of the instanton satisfies $\rho=\sqrt{y_{1}^{2}+\cdots y_{4}^{2}}$. The quantization procedures of the Lagrangian for the collective coordinates follows those in Ref. Specifically we need to assume that the moduli of the solution is time dependent. Thus, the gauge transformation also becomes time dependent as

$$
\begin{aligned}
\mathcal{A}_{M} & \rightarrow V\left(\mathcal{A}_{M}^{\mathrm{cl}}-i \partial_{M}\right) V^{-1}, \\
\mathcal{F}_{M N} & \rightarrow V \mathcal{F}_{M N}^{\mathrm{cl}} V^{-1}, \quad F_{0 M} \rightarrow V\left(\dot{X}^{\alpha} \partial_{\alpha} \mathcal{A}_{M}^{\mathrm{cl}}-D_{M}^{\mathrm{cl}} \Phi\right) V^{-1},
\end{aligned}
$$

The Lagrangian of the collective coordinates in such a moduli space takes the form as

$$
L=\frac{m_{X}}{2} \mathcal{G}_{r s} \dot{\mathcal{X}}^{s} \dot{\mathcal{X}}^{r}-U\left(\mathcal{X}^{s}\right)+\mathcal{O}\left(\lambda^{-1}\right)
$$

where $\mathcal{X}^{s}=\left\{X^{M}, a_{I}\right\}$. The the kinetic term in (C5) corresponds to the line element of the moduli space while the potential corresponds to the onshell action of the soliton adopting the time-dependent gauge transformation,

$$
S_{\mathrm{D} 8 / \overline{\mathrm{D} 8}}^{\mathrm{onshell}} \simeq S_{\mathrm{YM}+\mathrm{CS}}^{\mathrm{onshell}}=-\int d t U\left(\mathcal{X}^{s}\right)
$$

Using the solution (C2), the above integral is easy to calculate in the case of pure light flavors while it becomes quite difficult if the heavy flavor is involved. Without loss of generality, let us consider the large $\lambda$ limit followed by heavy mass limit of the heavy flavor. Hence the dimensionless quantized Hamiltonian corresponding to (C5) for the collective modes is calculated as

$$
\begin{aligned}
H & =M_{0}+H_{y}+H_{Z}+\mathcal{O}\left(\lambda^{-1} m_{H}^{0}\right), \\
H_{y} & =-\frac{1}{2 m_{y}} \sum_{I=1}^{4} \frac{\partial^{2}}{\partial y_{I}^{2}}+\frac{1}{2} m_{y} \omega_{y}^{2} \rho^{2}+\frac{\mathcal{Q}}{\rho^{2}}, \\
H_{Z} & =-\frac{1}{2 m_{Z}} \frac{\partial^{2}}{\partial Z^{2}}+\frac{1}{2} m_{Z} \omega_{Z}^{2} Z^{2},
\end{aligned}
$$

where

$$
\begin{aligned}
M_{0} & =8 \pi^{2} \kappa, \quad \omega_{Z}^{2}=\frac{2}{3}, \quad \omega_{\rho}^{2}=\frac{1}{6}, \quad \kappa=\frac{\lambda N_{c}}{216 \pi^{3}}, \\
\mathcal{Q} & =Q_{L}+Q_{H}, \quad Q_{L}=\frac{N_{c}}{40 \pi^{2} a}, \quad Q_{H}=\frac{N_{Q}}{8 \pi^{2} a}\left(\frac{N_{Q}}{3 N_{c}}-\frac{3}{4}\right) .
\end{aligned}
$$

The value of $\mathcal{Q}$ corresponds to the situation of a baryonic bound state consisting of $N_{Q}$ heavy flavored quarks. The eigenfunctions and mass spectrum of (C7) can be evaluated by solving its Schrodinger equation, respectively they are obtained as ${ }^{7}$

$$
\begin{aligned}
\psi\left(y_{I}\right)= & R(\rho) T^{(l)}\left(a_{I}\right), \\
R(\rho)= & e^{-\frac{m_{y} \omega_{\rho}}{2} \rho^{2}} \rho^{\tilde{l}} \text { Hypergeometric } 1 \\
& \times F_{1}\left(-n_{\rho}, \tilde{l}+2 ; m_{y} \omega_{\rho} \rho^{2}\right), \\
E\left(l, n_{\rho}, n_{z}\right)= & \omega_{\rho}\left(\tilde{l}+2 n_{\rho}+2\right) \\
= & \sqrt{\frac{(l+1)^{2}}{6}+\frac{640}{3} a^{2} \pi^{4} Q^{2}}+\frac{2\left(n_{\rho}+n_{z}\right)+2}{\sqrt{6}} .
\end{aligned}
$$

Notice that $T^{(l)}\left(a_{I}\right)$ satisfies $\nabla_{S^{3}}^{2} T^{(l)}=-l(l+2) T^{(l)}$ which is the function of the spherical part because $H_{y}$ can be written with the radial coordinate $\rho$ as

$$
H_{y}=-\frac{1}{2 m_{y}}\left[\frac{1}{\rho^{3}} \partial_{\rho}\left(\rho^{3} \partial_{\rho}\right)+\frac{1}{\rho^{2}}\left(\nabla_{S^{3}}^{2}-2 m_{y} \mathcal{Q}\right)\right]+\frac{1}{2} m_{y} \omega_{\rho}^{2} \rho^{2} \text {. }
$$

\footnotetext{
${ }^{6}$ In our notation, $\mathcal{A}$ is anti-Hermitian which means $\mathcal{A}^{\dagger}=-\mathcal{A}$.
}

\footnotetext{
${ }^{7} l$ and $\tilde{l}$ are related as $\tilde{l}=-1+\sqrt{(l+1)^{2}+2 m_{y} \mathcal{Q}}$.
} 


\section{APPENDIX D: EXPLICIT FORMULAS OF $\mathcal{L}_{1 / 2,0,-1 / 2}^{E, D, T}$ AND $\mathcal{L}_{\Psi}^{E, D, T}$}

Here we collect the explicit formulas of $\mathcal{L}_{1 / 2,0,-1 / 2}^{E, D, T}$ and $\mathcal{L}_{\Psi}^{E, D, T}$. For the exotic scalar glueball,

$$
\begin{aligned}
& \mathcal{L}_{1 / 2}^{E}=\frac{1}{M_{K K}}\left[-\frac{5}{4 M_{E}^{2}} \partial^{i} \partial^{j} G_{E} \mathcal{F}_{i k} \mathcal{F}_{j}^{k}+\frac{3}{16} G_{E} \mathcal{F}_{i j} \mathcal{F}^{i j}+\frac{5}{16 M_{E}^{2}} \partial^{2} G_{E} \mathcal{F}_{i j} \mathcal{F}^{i j}\right. \\
& \left.-\frac{5}{4 M_{E}^{2}} \partial^{i} \partial^{j} G_{E} \mathcal{F}_{i Z} \mathcal{F}_{j Z}-\frac{7}{8} \eta^{i j} G_{E} \mathcal{F}_{i Z} \mathcal{F}_{j Z}+\frac{5}{8 M_{E}^{2}} \partial^{2} G_{E} \eta^{i j} \mathcal{F}_{i Z} \mathcal{F}_{j Z}\right] \text {, } \\
& \mathcal{L}_{0}^{E}=\frac{1}{M_{E}^{2}}\left[\frac{20}{3} \partial^{k} G_{E} \eta^{i j} Z \mathcal{F}_{i k} \mathcal{F}_{j Z}-\frac{5}{2 M_{K K}} \partial^{0} \partial^{k} G_{E} \eta^{i j} \mathcal{F}_{i k} \mathcal{F}_{j 0}-\frac{5}{2 M_{K K}} \partial^{0} \partial^{i} G_{E} \mathcal{F}_{Z i} \mathcal{F}_{Z 0}\right], \\
& \mathcal{L}_{-1 / 2}^{E}=\frac{Z^{2}}{M_{K K}}\left[\frac{5}{16 M_{K K}^{2}} \partial^{i} \partial^{j} G_{E} \mathcal{F}_{i k} \mathcal{F}_{j}^{k}+\frac{15}{4 M_{E}^{2}} \partial^{i} \partial^{j} G_{E} \mathcal{F}_{i k} \mathcal{F}_{j}^{k}-\frac{5}{64 M_{K K}^{2}} \partial^{2} G_{E} \mathcal{F}_{i j} \mathcal{F}^{i j}\right. \\
& -\frac{3 M_{E}^{2}}{64 M_{K K}^{2}} G_{E} \mathcal{F}_{i j} \mathcal{F}^{i j}-\frac{35}{48} G_{E} \mathcal{F}_{i j} \mathcal{F}^{i j}-\frac{15}{16 M_{E}^{2}} \partial^{2} G_{E} \mathcal{F}_{i j} \mathcal{F}^{i j}+\frac{5}{16} \partial^{i} \partial^{j} G_{E} \mathcal{F}_{i Z} \mathcal{F}_{j Z} \\
& +\frac{25}{12} \frac{M_{K K}^{2}}{M_{E}^{2}} \partial^{i} \partial^{j} G_{E} \mathcal{F}_{i Z} \mathcal{F}_{j Z}-\frac{5}{32} \partial^{2} G_{E} \eta^{i j} \mathcal{F}_{i Z} \mathcal{F}_{j Z}+\frac{7}{32} M_{E}^{2} G_{E} \eta^{i j} \mathcal{F}_{i Z} \mathcal{F}_{j Z} \\
& \left.+\frac{9}{8} G_{E} M_{K K}^{2} \eta^{i j} \mathcal{F}_{i Z} \mathcal{F}_{j Z}-\frac{25}{24} \frac{M_{K K}^{2}}{M_{E}^{2}} \partial^{2} G_{E} \eta^{i j} \mathcal{F}_{i Z} \mathcal{F}_{j Z}\right]+\frac{1}{M_{E}^{2} M_{K K}}\left[\frac{5}{4} \partial^{i} \partial^{j} G_{E} \mathcal{F}_{i 0} \mathcal{F}_{j 0}\right. \\
& \left.-\frac{3}{8} M_{E}^{2} G_{E} \eta^{i j} \mathcal{F}_{i 0} \mathcal{F}_{j 0}-\frac{5}{8} \partial^{2} G_{E} \eta^{i j} \mathcal{F}_{i 0} \mathcal{F}_{j 0}-\frac{5}{4} \partial^{0} \partial^{0} G_{E} \eta^{i j} \mathcal{F}_{i 0} \mathcal{F}_{j 0}\right] \\
& +\frac{1}{M_{E}^{2} M_{K K}}\left[\frac{7}{8} G_{E} M_{E}^{2} \mathcal{F}_{Z 0} \mathcal{F}_{Z 0}-\frac{5}{8} \partial^{2} G_{E} \mathcal{F}_{Z 0} \mathcal{F}_{Z 0}-\frac{5}{4} \partial^{0} \partial^{0} G_{E} \mathcal{F}_{Z 0} \mathcal{F}_{Z 0}\right]-\frac{20}{3 M_{E}^{2}} Z \partial^{0} G_{E} \eta^{i j} \mathcal{F}_{j Z} \mathcal{F}_{i 0} \\
& \mathcal{L}_{\Psi}^{E}=-v^{2} \frac{\left(N_{f}+1\right)^{2}}{N_{f}^{2}}\left[-\frac{5}{12 M_{E}^{2} M_{K K}} \partial^{i} \partial^{j} G_{E} \Phi_{i}^{\dagger} \Phi_{j}+\frac{5}{24 M_{E}^{2} M_{K K}} \partial^{2} G_{E} \delta^{i j} \Phi_{i}^{\dagger} \Phi_{j}\right. \\
& \left.-\frac{5}{12 M_{K K}} G_{E} \Phi_{Z}^{\dagger} \Phi_{Z}+\frac{5}{24 M_{E}^{2} M_{K K}} \partial^{2} G_{E} \Phi_{Z}^{\dagger} \Phi_{Z}\right]
\end{aligned}
$$

For the dilatonic scalar glueball,

$$
\begin{aligned}
& \mathcal{L}_{1 / 2}^{D}=-\frac{\partial^{i} \partial^{j} G_{D}}{M_{D}^{2} M_{K K}} \mathcal{F}_{i k} \mathcal{F}_{j}^{k}+\frac{3 G_{D}}{4 M_{K K}} \mathcal{F}_{i j} \mathcal{F}^{i j}+\frac{\partial^{2} G_{D}}{4 M_{D}^{2} M_{K K}} \mathcal{F}_{i j} \mathcal{F}^{i j}-\frac{\partial^{i} \partial^{j} G_{D}}{M_{D}^{2} M_{K K}} \mathcal{F}_{i Z} \mathcal{F}_{j Z} \\
& +\frac{1}{2} G_{D} M_{K K}^{-1} \mathcal{F}_{Z i} \mathcal{F}_{Z}^{i}+\frac{\partial^{2} G_{D}}{2 M_{D}^{2} M_{K K}} \mathcal{F}_{Z i} \mathcal{F}_{Z}^{i} \\
& \mathcal{L}_{-1 / 2}^{D}=\frac{\partial^{i} \partial^{j} G_{D}}{4 M_{K K}^{3}} Z^{2} \mathcal{F}_{i k} \mathcal{F}_{j}^{k}+\frac{\partial^{i} \partial^{j} G_{D}}{3 M_{D}^{2} M_{K K}} Z^{2} \mathcal{F}_{i k} \mathcal{F}_{j}^{k}-\frac{\partial^{2} G_{D}}{16 M_{K K}^{3}} Z^{2} \mathcal{F}_{i j} \mathcal{F}^{i j}-\frac{3 G_{D} M_{D}^{2}}{16 M_{K K}^{3}} Z^{2} \mathcal{F}_{i j} \mathcal{F}^{i j}-\frac{G_{D}}{4 M_{K K}} Z^{2} \mathcal{F}_{i j} \mathcal{F}^{i j} \\
& -\frac{\partial^{2} G_{D}}{12 M_{D}^{2} M_{K K}} Z^{2} \mathcal{F}_{i j} \mathcal{F}^{i j}+\frac{\partial^{i} \partial^{j} G_{D}}{4 M_{K K}^{3}} Z^{2} \mathcal{F}_{i Z} \mathcal{F}_{j Z}-\frac{\partial^{i} \partial^{j} G_{D} Z^{2}}{M_{D}^{2} M_{K K}} \mathcal{F}_{i Z} \mathcal{F}_{j Z}-\frac{\partial^{2} G_{D} Z^{2}}{8 M_{K K}^{3}} \mathcal{F}_{Z i} \mathcal{F}_{Z}^{i}-\frac{G_{D} M_{D}^{2} Z^{2}}{8 M_{K K}^{3}} \mathcal{F}_{Z i} \mathcal{F}_{Z}^{i} \\
& +\frac{1}{2} G_{D} M_{K K}^{-1} Z^{2} \mathcal{F}_{Z i} \mathcal{F}_{Z}^{i}+\frac{\partial^{2} G_{D} Z^{2}}{2 M_{D}^{2} M_{K K}} \mathcal{F}_{Z i} \mathcal{F}_{Z}^{i}+\frac{\partial^{i} \partial^{j} G_{D}}{M_{D}^{2} M_{K K}} \mathcal{F}_{i 0} \mathcal{F}_{j 0}-\frac{3}{2} \frac{G_{D}}{M_{K K}} \mathcal{F}_{0 i} \mathcal{F}_{0}^{i}-\frac{\partial^{2} G_{D}}{2 M_{D}^{2} M_{K K}} \mathcal{F}_{0 i} \mathcal{F}_{0}^{i} \\
& -\frac{\partial^{0} \partial^{0} G_{D}}{M_{D}^{2} M_{K K}} \mathcal{F}_{0 i} \mathcal{F}_{0}^{i}-\frac{1}{2} G_{D} M_{K K}^{-1} \mathcal{F}_{0 Z}^{2}-\frac{\partial^{2} G_{D}}{2 M_{D}^{2} M_{K K}} \mathcal{F}_{0 Z}^{2}-\frac{\partial^{0} \partial^{0} G_{D}}{M_{D}^{2} M_{K K}} \mathcal{F}_{0 Z}^{2}, \mathcal{L}_{0}^{D}=0 \\
& \frac{\mathcal{L}_{\Psi}^{D}}{a \mathcal{C}_{D}}=v^{2} \frac{\left(N_{f}+1\right)^{2}}{N_{f}^{2}}\left[-\frac{\partial^{i} \partial^{j} G_{D}}{3 M_{D}^{2} M_{K K}} \Phi_{i}^{\dagger} \Phi_{j}+\frac{2 G_{D}}{3 M_{K K}} \eta^{i j} \Phi_{i}^{\dagger} \Phi_{j}+\frac{\partial^{2} G_{D}}{6 M_{D}^{2} M_{K K}} \eta^{i j} \Phi_{i}^{\dagger} \Phi_{j}+\frac{G_{D}}{3 M_{K K}} \Phi_{Z}^{\dagger} \Phi_{Z}+\frac{\partial^{2} G_{D}}{6 M_{D}^{2} M_{K K}} \Phi_{Z}^{\dagger} \Phi_{Z}\right] .
\end{aligned}
$$

For the tensor glueball, 


$$
\begin{aligned}
\mathcal{L}_{1 / 2}^{T}= & -\frac{T^{k l}}{M_{K K}} \eta^{i j} \mathcal{F}_{i k} \mathcal{F}_{j l}-\frac{T^{i j}}{M_{K K}} \mathcal{F}_{i Z} \mathcal{F}_{j Z}, \\
\mathcal{L}_{0}^{T}= & -\frac{2 T^{0 k}}{M_{K K}} \eta^{i j} \mathcal{F}_{i k} \mathcal{F}_{j 0}-\frac{2 T^{0 i}}{M_{K K}} \mathcal{F}_{Z i} \mathcal{F}_{Z 0}, \\
\mathcal{L}_{-1 / 2}^{T}= & \frac{T^{k l} \eta^{i j}}{3 M_{K K}} Z^{2} \mathcal{F}_{i k} \mathcal{F}_{j l}+\frac{M_{T}^{2} T^{k l} \eta^{i j}}{4 M_{K K}^{3}} Z^{2} \mathcal{F}_{i k} \mathcal{F}_{j l} \\
& -M_{K K} T^{i j} Z^{2} \mathcal{F}_{i Z} \mathcal{F}_{j Z}+\frac{M_{T}^{2}}{4 M_{K K}} T^{i j} Z^{2} \mathcal{F}_{i Z} \mathcal{F}_{j Z} \\
& +\frac{T^{i j}}{M_{K K}} \mathcal{F}_{i 0} \mathcal{F}_{j 0}-\frac{T^{00}}{M_{K K}} \eta^{i j} \mathcal{F}_{i 0} \mathcal{F}_{j 0}-\frac{T^{00}}{M_{K K}} \mathcal{F}_{Z 0} \mathcal{F}_{Z 0}, \\
\mathcal{L}_{\Psi}^{T}= & -\frac{\left(N_{f}+1\right)^{2}}{3 N_{f}^{2} M_{K K}} v^{2} T^{i j} \Phi_{i}^{\dagger} \Phi_{i} .
\end{aligned}
$$

We assume the glueball field is onshell so that $G_{E, D, T}$ could be chosen as $G_{E, D, T}=\frac{1}{2}\left(e^{-i M_{E, D, T} t}+\right.$ c.c $)$ in the rest frame of the glueball; hence, we have $\partial^{i} G_{E, D, T}=0$, $\partial_{\mu} \partial^{\mu} G_{E, D, T}=M_{E, D, T}^{2} G_{E, D, T}$ which could greatly simplify (D1) (D2) (D3). Since the $\mathcal{L}_{\Psi}^{E, D, T}$ refers to the mass term of the HL field, the mass of the heavy quarks $m_{H}$ must be related to the separation of the flavor branes i.e., the VEV of $\Psi$. In the heavy quark limit, the explicit relation is given as [24-26],

$$
\begin{aligned}
m_{H} & =\frac{1}{\pi l_{s}^{2}} \lim _{z_{H} \rightarrow \infty} \int_{0}^{z_{H}} d z \sqrt{-g_{00} g_{z z}}, \\
& \simeq \frac{1}{\pi l_{s}^{2}} U_{K K}^{1 / 3} z_{H}^{2 / 3}+\mathcal{O}\left(z_{H}^{0}\right) . \\
& \equiv \frac{1}{\sqrt{6}} \frac{N_{f}+1}{N_{f}} v
\end{aligned}
$$

where $z_{H}$ refers to the position $U=U_{H}$. Then we further collect the terms of $\mathcal{O}\left(m_{H}^{2}\right)$ and $\mathcal{O}\left(m_{H}\right)$ then integral out the part of $z$, it finally leads to the formulas in (3.4).
[1] H. Fritzsch and M. Gell-Mann, Current algebra: Quarks and what else, eConf C720906V2, 135 (1972).

[2] H. Fritzsch and P. Minkowski, $\Psi$ resonances, gluons and the Zweig rule, Nuovo Cimento A 30, 393 (1975).

[3] R. Jaffe and K. Johnson, Unconventional states of confined quarks and gluons, Phys. Lett. 60B, 201 (1976).

[4] C. J. Morningstar and M. J. Peardon, The Glueball spectrum from an anisotropic lattice study, Phys. Rev. D 60, 034509 (1999).

[5] Y. Chen, A. Alexandru, S. Dong, T. Draper, I. Horvath et al., Glueball spectrum and matrix elements on anisotropic lattices, Phys. Rev. D 73, 014516 (2006).

[6] H. B. Meyer and M. J. Teper, Glueball Regge trajectories and the pomeron: A Lattice study, Phys. Lett. B 605, 344 (2005).

[7] E. Gregory, A. Irving, B. Lucini, C. McNeile, A. Rago, C. Richards, and E. Rinaldi, Towards the glueball spectrum from unquenched lattice QCD, J. High Energy Phys. 10 (2012) 170.

[8] Y. K. Hsiao and C. Q. Geng, Identifying Glueball at $3.02 \mathrm{GeV}$ in baryonic B decays, Phys. Lett. B 727, 168 (2013).

[9] X.-G. He and T.-C. Yuan, Glueball production via gluonic penguin B decays, Eur. Phys. J. C 75, 136 (2015).

[10] X.-W. Kang, T. Luo, Y. Zhang, L.-Y. Dai, and C. Wang, Semileptonic B and Bs decays involving scalar and axialvector mesons, Eur. Phys. J. C 78, 909 (2018).

[11] K. Hashimoto, C.-I. Tan, and S. Terashima, Glueball decay in holographic QCD, Phys. Rev. D 77, 086001 (2008).
[12] E. Witten, Anti-de Sitter space, thermal phase transition, and confinement in gauge theories, Adv. Theor. Math. Phys. 2, 505 (1998).

[13] T. Sakai and S. Sugimoto, Low energy hadron physics in holographic QCD, Prog. Theor. Phys. 113, 843 (2005).

[14] F. Brünner, D. Parganlija, and A. Rebhan, Glueball decay rates in the Witten-Sakai-Sugimoto model, Phys. Rev. D 91, 106002 (2015); Erratum, Phys. Rev. D93, 109903(E) (2016).

[15] S.-w. Li, The interaction of glueball and heavy-light flavoured meson in holographic QCD, arXiv:1809.10379.

[16] F. Brünner, J. Leutgeb, and A. Rebhan, A broad pseudovector glueball from holographic QCD, Phys. Lett. B 788, 431 (2019).

[17] N. R. Constable and R. C. Myers, Spin-two glueballs, positive energy theorems and the AdS/CFT correspondence, J. High Energy Phys. 10 (1999) 037.

[18] R. C. Brower, S. D. Mathur, and C.-I. Tan, Glueball spectrum for QCD from AdS supergravity duality, Nucl. Phys. B587, 249 (2000).

[19] S.-w. Li, Glueball-baryon interactions in holographic QCD, Phys. Lett. B 773, 142 (2017).

[20] E. Witten, Baryons and branes in anti-de Sitter space, J. High Energy Phys. 07 (1998) 006.

[21] D. Tong, TASI lectures on solitons: Instantons, monopoles, vortices and kinks, arXiv:hep-th/0509216.

[22] H. Hata, T. Sakai, S. Sugimoto, and S. Yamato, Baryons from instantons in holographic QCD, Prog. Theor. Phys. 117, 1157 (2007).

[23] Y. Liu and I. Zahed, Holographic heavy-light chiral effective action, Phys. Rev. D 95, 056022 (2017).

[24] Y. Liu and I. Zahed, Heavy-light mesons in chiral AdS/ QCD, Phys. Lett. B 769, 314 (2017). 
[25] Y. Liu and I. Zahed, Heavy baryons and their exotics from instantons in holographic QCD, Phys. Rev. D 95, 116012 (2017).

[26] S.-w. Li, Holographic heavy-baryons in the Witten-SakaiSugimoto model with the D0-D4 background, Phys. Rev. D 96, 106018 (2017).
[27] K. Becker, M. Becker, and J. H. Schwarz, String theory and M-theory, A Modern Introduction (Cambridge University Press, Cambridge, England, 2007).

[28] R. C. Myers, Dielectric-Branes, J. High Energy Phys. 12 (1999) 022. 